\title{
Análisis descriptivo de las concepciones sobre historia de las ciencias en profesores en formación inicial
}

\author{
Descriptive analysis of conceptions of the history of science in teachers \\ in initial training
}

\section{Análise descritiva das concepções sobre a história da ciência em professores em formação inicial}

\author{
Henry Giovany Cabrera Castilloa," | Stefania Durán Piambab | Mario Quintanilla Gatica \\ ${ }^{a}$ http://orcid.org/0000-0003-4476-4427 Universidad del Valle, Cali, Colombia \\ b Universidad del Valle, Cali, Colombia \\ c Pontificia Universidad Católica de Chile, Santiago, Chile
}

- Fecha de recepción: 2017-09-19

- Fecha concepto de evaluación: 2019-09-03

- Fecha de aprobación: 2019-09-29 http://dx.doi.org/ | 0.22335/rlct.v I I i2.482
Para citar este artículo / To reference this article / Para citar este artigo: Cabrera Castillo, H. G., Durán Piamba, S., \& Quintanilla Gatica, M. (2019). Análisis descriptivo de las concepciones sobre historia de las ciencias en profesores en formación inicial. Revista Logos Ciencia \& Tecnología, I I (2), 34-45. http://dx.doi.org/ I0.22335/rlct.v I Ii2.482

\section{RESUMEN}

Este artículo tuvo como objetivo identificar las concepciones sobre historia de la ciencia que tienen los profesores en formación inicial. Se utilizó una metodología cualitativo-descriptiva. Los datos fueron obtenidos a partir de un cuestionario inicial, estructurado por preguntas abiertas y la construcción de un documento escrito final aplicado a veinticinco participantes de un programa de licenciatura en la Universidad delValle. Los resultados de la propuesta se obtuvieron a partir de un análisis descrito de tres categorías (visión de la historia de las ciencias, función de la historia de las ciencias en la enseñanza y producción de la ciencia) que permitió determinar que las concepciones sobre historia de las ciencias que los profesores en formación inicial han elaborado corresponden a las visiones anacrónica y diacrónica, funciones como actividad social, recurso y disciplinar, y producción de conocimiento. Como conclusión, podemos establecer que es necesario implementar propuestas didácticas direccionadas hacia el uso de la historia de las ciencias con el propósito de favorecer en ellos el reconocimiento, la reflexión y el uso de componentes históricos en sus prácticas educativas.

Palabras clave: concepciones de la ciencia, didáctica de las ciencias, formación inicial de profesores, historia de las ciencias 


\section{SUMMARY}

The purpose of this article was to identify the conceptions of the history of science held by teachers in initial training. A qualitative-descriptive methodology was used. The data were obtained from an initial questionnaire, structured by open-ended questions and the construction of a final written document applied to twenty-five participants on a degree program at Universidad delValle. The results of the proposal were obtained from a descriptive analysis of three categories (vision of the history of the sciences, function of the history of the sciences in teaching and production of science) which made it possible to determine that the conceptions of the history of the sciences that teachers in initial training have elaborated correspond to anachronistic and diachronic visions, functions such as social activity, resource and disciplinary, and production of knowledge. As a conclusion, we can establish that it is necessary to implement didactic proposals directed toward the use of the history of science so as to promote in teachers the recognition, reflection, and use of historical components in their educational practices.

Keywords: Conceptions of science, didactics of science, initial teacher-training, history of science

\section{SUMÁRIO}

Este artigo teve como objetivo identificar as concepções sobre a história da ciência que os professores têm durante a formação inicial. Foi utilizada uma metodologia qualitativa-descritiva. Os dados foram obtidos a partir de um questionário inicial estruturado em perguntas abertas e a construção de um documento final escrito aplicado a vinte e cinco participantes de um programa de graduação na Universidad del Valle. Os resultados da proposta foram obtidos a partir de uma análise descrita de três categorias (visão da história da ciência, função da história da ciência no ensino e produção da ciência) que permitiu determinar que as concepções sobre a história das ciências que os professores em formação inicial elaboraram correspondem às visões anacrônicas e diacrônicas, funções como atividade social, recurso e disciplina e produção de conhecimento. Em conclusão, podemos estabelecer que é necessário implementar propostas didáticas voltadas para o uso da história da ciência, a fim de favorecer o reconhecimento, a reflexão e o uso de componentes históricos em suas práticas educativas.

Palavras-chave: Concepções da ciência, ensino de ciências, formação inicial de professores, história da ciência

La historia y filosofía de las ciencias en la formación de profesores de ciencias naturales ha sido utilizada para identificar preguntas, instrumentos, experimentos $y$ explicaciones de los científicos sobre los fenómenos para recontextualizarlos en las prácticas educativas actuales. Estas metadisciplinas han permitido evidenciar la complejidad de la actividad humana, inspirar nuevas estrategias para la presentación de problemas complejos, diseñar e implementar herramientas para la enseñanza y el aprendizaje de las ciencias y reconstruir textos históricos para incorporarlos en las prácticas de la clase de ciencias (Cabrera, 2016; Izquierdo, García-Martínez, Quintanilla, \& Adúriz-Bravo, 2016).

Los hallazgos obtenidos de las investigaciones anteriores recobran importancia en el diseño de propuestas didácticas.
Dichas propuestas tendrán como objetivos determinar la importancia del uso de textos científico-históricos escritos por los científicos en una época determinada, elaborar estudios de caso histórico-científico desde una mirada educativa e identificar las concepciones sobre historia de las ciencias que tienen los profesores de ciencias naturales en formación inicial (Cabrera, 2012). En este orden de ideas, el siguiente estudio se enfocó principalmente en el análisis descriptivo del último objetivo mencionado, por lo que se consideró necesario reconocer las concepciones sobre historia de las ciencias con las que inician $y$ finalizan los futuros profesores de ciencias.

Dichas concepciones permitirán identificar dificultades en la conceptualización e interpretación del desarrollo de la ciencia, en la apropiación de conocimiento e igualmente 
en las diferentes visiones que tienen sobre las ciencias (Fernández, Gil, Carrascosa, Cachapuz, \& Praia, 2002). Con el estudio de estas concepciones, se puede determinar qué funciones le atribuyen los profesores en formación inicial, es decir, si la historia de las ciencias es utilizada para el diseño de materiales, para complementar la formación de los profesores, en la formulación de preguntas, en la actividad experimental o en la selección de contenidos (Cuéllar, Quintanilla, \& Camacho, 2008; Heering, \& Wittje, 20I2; Zuluaga, 20I2).

Otro aspecto que se puede extraer es lo correspondiente a la manera como se interpreta la producción de conocimiento científico en los contextos actuales, por ejemplo, en la aplicación que se le da al conocimiento científico en procesos industriales, farmacéuticos, ambientales y de salud, y su vínculo con el desarrollo vertiginoso y exponencial de la tecnología. De acuerdo con la anterior, el objetivo de este trabajo consiste en identificar las concepciones sobre historia de las ciencias que posee un grupo de profesores de ciencias naturales en formación inicial, antes y después de la aplicación de una propuesta didáctica.

\section{Marco teórico}

Los aportes de la historia de las ciencias a la educación en ciencias están asociados a su incorporación en el diseño de unidades didácticas y en la formulación de situaciones problemáticas para la educación básica y media. Por ejemplo, Quintanilla y Merino (2008) avanzaron hacia la elaboración de materiales a partir de los aportes de la historia, y Muñoz, Valencia y Cabrera (2017) diseñaron situaciones científicas escolares problematizadoras, a partir del análisis de un experimento histórico elaborado por Robert Boyle (|627-|69|).

Otra tendencia corresponde a la incorporación de la historia de las ciencias en la formación de profesorado en ejercicio. En este caso, tenemos a Álvarez (2006), quien propuso estrategias que permitían incorporar a su desarrollo profesional. Es importante visualizar esta tendencia en la formación inicial de profesores de ciencias. Para este fin, se identificaron estudios orientados hacia el desarrollo de una propuesta que permitió el cambio de concepciones en profesores de educación parvularia en la enseñanza de la ciencia a través de la historia de las ciencias (Camacho, Cuéllar, Quintanilla, \& Orellana, 2006). En consonancia con lo expresado en la última tendencia, este artículo considera fundamental continuar explorando y conceptualizando las concepciones y la importancia de la historia de las ciencias que tienen los profesores en formación inicial.

\section{Importancia de la historia de la ciencia en la} formación inicial del profesorado de ciencias

La historia de las ciencias permite identificar y caracterizar los modelos teóricos y las metodologías de las disciplinas científicas, relacionar el conocimiento científico con los problemas y finalidades, la cultura y los valores del contexto (Matthews, 1994). Cuando esta metadiscipina se lleva a la educación en ciencias, permite que tanto profesores como estudiantes expresen y estructuren sus ideas acerca de la ciencia, comprendan que los modelos científicos son modificables y que, por tanto, el conocimiento científico actual es susceptible de ser evaluado y transformado.

En lo que corresponde a la formación inicial de profesores, la importancia que tiene el uso de la historia de las ciencias radica en comprender que es una oportunidad para reflexionar sobre el pensamiento científico y promover la reflexión sobre fenómenos químicos, físicos, biológicos y ambientales en el aula. Igualmente, permite lograr el cambio de visiones deformadas que se hayan construido en su proceso formativo y contribuir en el cambio de las concepciones de los futuros profesores sobre la ciencia (Quintanilla, Izquierdo, \& Adúriz-Bravo, 2005; Solsona, 1997).

\section{Concepciones sobre historia de la ciencia de los profesores en formación inicial}

Los profesores en formación inicial han logrado construir diversas concepciones del mundo y de las ciencias, que han sido adquiridas con sus experiencias en la construcción de conocimiento en la escuela y luego en la formación profesional. Algunos estudios se han orientado hacia las concepciones que tienen estos profesores sobre el uso de modelos en la formación docente, la resolución de problemas científicos escolares y las imágenes o representaciones que ellos han construido sobre la historia de las ciencias (Cabrera, 20I5; Justi, 2006).

En función del último aspecto mencionado, Quintanilla, Astroza, De la Fuente, Camacho y Cuéllar (2006) exploraron las concepciones que tienen los futuros profesores sobre las metaciencias, dado que estas configuran un marco 
conceptual importante disciplinar, ya que promueven diferentes imágenes sobre conocimiento científico y su enseñanza. Este trabajo aporta elementos teóricos que justifican la importancia de incorporar los componentes filosóficos e históricos en los diferentes programas de ciencias.

Asimismo, Camacho et al. (2006), con el propósito de identificar y caracterizar las concepciones de algunas profesoras de educación parvularia, desarrollaron una propuesta que incorporó el uso de la historia de las ciencias como componente metacientífico, con el uso de un episodio histórico sobre la discontinuidad de la materia, ya que suscita reflexiones sobre la actividad científica y permite contribuir en la construcción de una imagen de la ciencia.

Para finalizar, los trabajos anteriores permiten destacar dos aspectos fundamentales. El primero corresponde a la persistencia de concepciones dogmáticas, utilitaristas e instrumentalistas de las ciencias, lo que conlleva producir un desinterés de los futuros profesores de ciencia, ya que la relacionan con acontecimientos lineales y desconectados de la práctica. En otras palabras, se ha perpetuado la concepción heredada de la ciencia en la que se cataloga a los científicos como los creadores de teorías y poseedores de la verdad absoluta.

En segundo lugar, es fundamental continuar con estudios que permitan identificar y caracterizar las concepciones que tienen los profesores en formación inicial sobre historia de las ciencias, ya que estos pueden servir como orientación para el diseño y la integración de esta metaciencia en la formación del profesorado. Por ejemplo, Quintanilla et al. (2005) lo destacan como uno de los aspectos iniciales para incorporar la historia de las ciencias en la formación docente inicial, y vincular la solución de problemas "actuales" de las teorías científicas, que conlleven el diseño y la implementación de propuestas didácticas.

\section{Método: planteamientos} procedimentales del estudio

Dentro del curso Historia y Enseñanza de las Ciencias se estableció como finalidad estudiar la historia de las ciencias como marco conceptual para la investigación y la reflexión, así como para orientar el proceso de enseñanza-aprendizaje y evaluación de las ciencias en la formación educativa científica. En este sentido, adquirío importancia el propósito de identificar las concepciones que poseía un grupo de futuros profesores de ciencias, antes y después de la aplicación de una propuesta didáctica.

El enfoque metodológico del estudio fue el cualitativo, de tipo descriptivo e interpretativo (Flick, 2004; Hernández, Fernández, \& Baptista, 2008; Latorre, del Rinón, \& Arnal, 1996), ya que se enfoca en las ideas elaboradas por los participantes antes y después de interactuar con nuevas experiencias académicas desarrolladas a lo largo de un periodo determinado. Los participantes fueron veinticinco estudiantes de la Licenciatura en Educación Básica con Énfasis en Ciencias Naturales y Educación Ambiental de la Universidad del Valle (Cali, Colombia). Ellos firmaron un consentimiento por escrito y aceptaron el alcance de la investigación.

En concordancia con el enfoque cualitativo seleccionado y basados en los planteamientos sugeridos por Quintanilla et al. (2006), en el inicio del curso se aplicó un cuestionario con tres preguntas abiertas en el que los participantes debían: (a) elaborar un texto en el que determinaran la función que debe desempeñar la historia de las ciencias en la enseñanza de las ciencias; (b) establecer una relación entre historia de las ciencias, filosofía de las ciencias y enseñanza de las ciencias; $y$ (c) describir cómo la ciencia se ha logrado consolidar a lo largo del tiempo.

Al culminar el curso, se les solicitó a los participantes que entregaran un documento escrito que diera respuesta al propósito del programa y que tuviera como eje un caso de estudio histórico de biología, química o física. La elaboración de dicho documento estuvo basada en los elementos de Cabrera (2016) y Holton (1995) (tabla I). A efectos del estudio que se está presentando, la interpretación se centró solo en la introducción, la importancia histórica y actual del caso, el análisis del textos científico-históricos y los aportes a la enseñanza.

Para el procesamiento analítico de la información, se consideraron los planteamientos de Hernández et al. (2008) y Flick (2004), y las tres fases propuestas por Bardin (2002): preanálisis correspondiente a la lectura previa, selección y transcripción con un procesador de texto (Word) de los cuestionarios completamente resueltos por los participantes. Estos cuestionarios constituyeron el corpus de documentos que fueron sometidos a la descripción e interpretación.

La explotación del material se dio con la desfragmentación en unidades de análisis (UA) (oraciones completas) 
Tabla I. Elementos para la elaboración del trabajo final del curso Historia y Enseñanza de las Ciencias

\begin{tabular}{|c|c|}
\hline Elementos & Descripción \\
\hline Introducción & Debe cumplir tres funciones, interesar al lector, presentar un tema y bosquejar un plan. \\
\hline Importancia histórica y actual del concepto seleccionado & $\begin{array}{l}\text { Presentación de los aspectos fundamentales tanto del contexto histórico, como de } \\
\text { actualidad en la cual se desarrolla el concepto. }\end{array}$ \\
\hline Biografía del Científico o Científica seleccionada & $\begin{array}{l}\text { Descripción de los principales aspectos relacionados con su contexto, político, económi- } \\
\text { co, social y familiar de uno de los científicos que realizo aportes para la construcción } \\
\text { del concepto. }\end{array}$ \\
\hline Descripción bibliográfica del TCH & $\begin{array}{l}\text { Descripción de la estructura (título, editorial, año, revista, volumen, número y páginas) } \\
\text { del } T C H \text {. }\end{array}$ \\
\hline Análisis del $T C H$ & $\begin{array}{l}\text { Se tuvo en cuenta el análisis histórico-critico como un proceso de "dialogo" entre el } \\
\text { estudiante y el científico o científica que describió el } T C H \text {. }\end{array}$ \\
\hline Aportes a la enseñanza en base al análisis & $\begin{array}{l}\text { Teniendo en cuenta el proceso de análisis y sus hallazgos se diseñaron actividades } \\
\text { orientadas hacia la enseñanza de concepto }\end{array}$ \\
\hline Referencias bibliográficas & Inclusión de los diferentes investigadores que elaboraron la literatura revisada. \\
\hline
\end{tabular}

relevantes y significativas, que se asociaban a los códigos, subcategorías y categorías predeterminadas, conceptualizadas y adaptadas a partir de la revisión de la investigación de Quintanilla et al. (2006) y tratando de expresar los datos en forma de conceptos o ideas que estén en concordancia con el propósito de la investigación (Gibbs, 2012) (tabla 2). Este proceso se complementó con el uso del software Atlas.ti versión 7.5.16 para determinar las unidades de análisis (UA) que estructuraron los hallazgos.

El tratamiento y la interpretación de los resultados obtenidos consistió en la inferencia realizada a partir de las UA significativas seleccionadas y organizadas por medio de redes que daban información relevante para el propósito del estudio.

La propuesta didáctica del curso se dividió en tres momentos: primero, para el diseño del curso, se programaron actividades para las quince sesiones que duraba el periodo académico. Se consideraron aspectos conceptuales sobre la filosofía de la ciencia, las perspectivas sobre historia de la ciencia y las relaciones entre ellas, y se estudiaron casos históricos desde una mirada educativa y didáctica (Boido, \& Lombardi, 20 I2a; Echeverría, 1998; Hernández, 20II; Kragh, 1990; Valencia, Muñoz, \& Cabrera, 2014).

Para el segundo momento, se desarrollaron sesiones con la finalidad de considerar las expectativas de los estudiantes en relación con el contenido, la metodología y los criterios de evaluación de este. Sumado a ello, se aplicó el cuestionario diseñado con el propósito de identificar las concepciones que los profesores en formación inicial tenían sobre historia de la ciencia.
En otras sesiones, se desarrollaron las siguientes fases: lectura previa de investigaciones y desarrollos teóricos reportados en capítulos de libros y artículos de revistas; socialización de ideas fundamentales de las lecturas asignadas para la sesión con diferentes modalidades de trabajo grupal (seminario, debate, torbellino de ideas); $y$ conclusión con la que el profesor cerraba y sintetizaba la sesión. Participación de tres invitados quienes desarrollaron conferencias orientadas hacia la presentación de algunos estudios de caso histórico desde una mirada educativa y didáctica (la experimentación en Newton, el experimento $V$ de Boyle y Homeostasis).

Igualmente, los estudiantes desarrollaron dos talleres. EI primero estuvo orientado hacia la búsqueda y selección del caso histórico y la indagación del o los textos científicohistóricos (e.g., artículos, cuadernos de notas delaboratorio, bitácoras, discursos, capítulos y libros, conferencias, entrevistas) escritos por algún científico en un periodo determinado. El segundo consistió en el análisis de libros de texto universitarios de ciencias naturales en los que se identificaron aspectos inherentes a la historia de la ciencia. Finalmente, para la evaluación del curso, los participantes entregaron un documento escrito en atención a las indicaciones y estructura que aparece en la tabla $\mathrm{I}$.

\section{Resultados y discusión}

A partir de la información obtenida con los cuestionarios iniciales y los trabajos finales entregados por los participantes del estudio, logramos identificar las UA relacionadas con la historia de la ciencia, lo que contribuye al 
Tabla 2. Compilado de categorías, subcategorías y códigos estado final

Visiones sobre historia de la ciencia: Se incluyen unidades de análisis correspondientes a las imágenes construidas sobre la historia de las ciencias por parte de los PCNFI a través de su formación.
Anacrónica: Se incluyen unidades de An de aquellas ideas de los PCNFI que interpretan el pasado de las ciencias desde una perspectiva y lenguaje actual y descontextualizado con la época que corresponde (Izquierdo et al., 2016).

Acumulativa: Se incluyen unidades de análisis correspondientes a frases que hacen referencia a la construcción de la ciencia como un proceso lineal y simplista en el cual únicamente importa la fecha en la cual se realizó un descubrimiento e ignora los problemas o hechos científicos que permitieron la construcción de las ciencias y confiere la construcción de las ciencias exclusivamente a los aportes de un científico cuyos resultados son suficientes para verificar o falsear hipótesis (Fernández et al., 2002).

Diacrónica: Se incluyen unidades Valoración de Contexto: Se incluyen unidades de análisis de ideas de análisis de aquellas ideas de los que destacan la influencia de las expectativas sociales y culturaPCNFI que interpretan el pasado de les en la construcción de las ciencias y confiere la construcción las ciencias desde una perspectiva y de las ciencias y las acciones provienen de los aportes de varios lenguaje contextualizado en la época científicos y científicas (Fernández et al., 2002; Quintanilla, 2006b) que corresponde (Izquierdo, 20I4; Quintanilla, 2006a).

Función de la $\mathrm{HC}$ en la enseñanza y aprendizaje de las ciencias: Se incluyen unidades de análisis que corresponde a la intención y utilidad de la historia de las ciencias al proceso de enseñanza y aprendizaje de las ciencias que le asignan los PCNFI.
Disciplinar: Se incluyen unidades de análisis de ideas que destacan aspectos relacionados con el contenido conceptual derivados de la historia de las ciencias.
Conceptuales: Se incluyen unidades de análisis que involucra definiciones, modelos, teorías y representaciones a nivel conceptual que los PCNFI extraen de la historia de las ciencias.

Actividad experimental: se incluyen unidades de análisis en los que se destaca el uso de la experimentación, instrumentos de laboratorio, experimentos históricos, fenómenos científicos y procedimientos materiales

Actividad social: Se incluyen unida- Formación de profesores: Se incluyen unidades de análisis de des de análisis que hacen referencia elementos que pueden extraerse de la historia de las ciencias a la aplicabilidad que tiene la historia para integrar, mejorar y fortalecer la formación de los profesode las ciencias en los procesos donde res de ciencias. participan diversos colectivos.

Práctica educativa:Se incluyen unidades de análisis donde se destaca elementos de la enseñanza, aprendizaje y evaluación en los procesos de construcción de conocimiento científico escolar.

Recurso: Se incluyen unidades de Uso de materiales: Se incluyen unidades de análisis de frases en las análisis de frases en las que los PCNFI determinan a partir de la historia de las ciencias el mejoramiento, perfección, adecuación, implementación de materiales didácticos en la enseñanza y aprendizaje de las ciencias. aprendizaje de las ciencias.

Formulación de preguntas: Se incluyen unidades de análisis correspondientes a las diferentes preguntas que formulan los PCNFI en el diseño de actividades para la enseñanza de la temática analizada proveniente del análisis histórico.

Desarrollo: Se incluyen unidades de análisis circunscritas al proceso mediante el cual las ciencias resuelven problemáticas e interactúa con Aplicación: Se incluyen unidades de análisis correspondientes al uso y mejoramiento de instrumentos, artefactos, técnicas y modelos que se han producido en las ciencias. Se incluyen unidades de análisis correspondientes a la interpretación que tienen los PCNFI sobre la producción y desarrollo del conocimiento científico como una actividad humana. reconocimiento de las concepciones iniciales y finales sobre esa metadisciplina. En la tabla 3 , se presentan las UA desde el punto de vista de la frecuencia y el porcentaje de participación en las diferentes categorías, subcategorías y códigos en fase inicial y fase final.

En cuanto a la categoría Visión de la HC y, específicamente, la subcategoría Anacrónica, identificamos que los profesores en formación inicial incluyeron en sus respuestas 31 UA asociadas al código (acumulativa), de las que el 67,7 \% las utilizaron al inicio y el $32,3 \%$ al finalizar el proceso acaecido durante el semestre. Algunas de las UA fueron las siguientes:"La idea de explicar la naturaleza a través de la ciencia y verla como exacta y verdadera", y "La historia de las ciencias y la historia en general en demasiadas ocasiones han sido un punto de partida y un poderoso instrumento para quienes se interesan en legitimar el presente".

Los resultados del cuestionario coinciden con los planteamientos de Urzúa y Rodríguez-Pineda (20I5), ya que la visión anacrónica o el perfil epistemológico positivista como ellos la denominan fue el dominante porque conciben la ciencia como empírica. 
Tabla 3. Comparación de frecuencia y porcentajes por código

\begin{tabular}{|c|c|c|c|c|c|c|c|}
\hline \multirow[b]{2}{*}{ Categoría } & \multirow[b]{2}{*}{ Subcategoría } & \multirow[b]{2}{*}{ Código } & \multicolumn{2}{|c|}{ Inicial } & \multicolumn{2}{|r|}{ Final } & \multirow[b]{2}{*}{$\begin{array}{c}\text { Total citas } \\
\text { participativas }\end{array}$} \\
\hline & & & Frecuencia & $\begin{array}{c}\text { Porcentaje de } \\
\text { participación } \%\end{array}$ & Frecuencia & $\begin{array}{c}\text { Porcentaje de } \\
\text { participación } \%\end{array}$ & \\
\hline \multirow{2}{*}{$\begin{array}{l}\text { Visión de } \\
\text { la HC }\end{array}$} & Anacrónica & Acumulativa & 21 & 67,7 & 10 & 32,3 & 31 \\
\hline & Diacrónica & Valoración del Contexto & 19 & $4 I, 3$ & 27 & 58,7 & 46 \\
\hline \multirow{6}{*}{$\begin{array}{l}\text { Función } \\
\text { de la HC }\end{array}$} & \multirow{2}{*}{ Disciplinar } & Conceptuales & 14 & 53,8 & 12 & 46,2 & 26 \\
\hline & & Actividad Experimental & I & 7,1 & 13 & 92,9 & 14 \\
\hline & \multirow{2}{*}{ Actividad social } & Práctica Educativa & 4 & 13,8 & 25 & 86,2 & 29 \\
\hline & & Formación de Profesores & 17 & 58,6 & 12 & $4 I, 4$ & 29 \\
\hline & \multirow[b]{2}{*}{ Recurso } & Uso de Materiales & 3 & 13,0 & 20 & 87,0 & 23 \\
\hline & & $\begin{array}{l}\text { Formulación } \\
\text { de Preguntas }\end{array}$ & 0 & 0,0 & 112 & 100,0 & 112 \\
\hline $\begin{array}{l}\text { Aplicación } \\
\text { de la HC }\end{array}$ & Desarrollo & Aplicación & 8 & 50,0 & 8 & 50,0 & 16 \\
\hline
\end{tabular}

En la subcategoría Diacrónica, los profesores en inicial emitieron 46 UA relacionadas con el código valoración del contexto, de ellas, el 4I,3 \% las expresaron al inicio y el $58,7 \%$ al final. Algunas de las UA fueron: "La ciencia está sometida de cierta manera a unas particularidades, como la época, la cultura, las creencias y los métodos" y "Es fundamental el contexto histórico en el que se desarrollaron sus planteamientos y el impacto que generó en los científicos de la edad moderna".

De acuerdo con los resultados, se logra establecer que inicialmente los futuros profesores presentan una visión acumulativa y, después del proceso académico, empezaron a valorar el contexto. Sin embargo, algunos estudiantes continúan representando la ciencia como exacta, verdadera y legítima. Esto se da porque los discursos hegemónicos que prevalecen en las clases y en los libros de texto universitario son difíciles de cambiar (Borrachero, Brígido, \& Costillo, 20II). Es necesario avanzar hacia propuestas que presenten la importancia de valorar la ciencia como una actividad humana que responda a particularidades de la época, evalúe diversas perspectivas y reconozca los aspectos culturales, sociales y las creencias situadas en un contexto determinado en el que se evidencian las transformaciones de una sociedad (Padilla, Furió, \& Azcona, 2005; Quintanilla, 2006a).

En la medida en que se diseñen, desarrollen y evalúen propuestas tanto en asignaturas del componente didáctico como disciplinar, en las que se destacan y valoran las perspectivas de los diferentes científicos como integrantes y partícipes activos de la sociedad, se avanzará paulatinamente hacia la superación de las ideas simplistas de la ciencia y el papel individualista del científico (Gallego, \& Gallego, 2007).

En cuanto a la categoría Función de la $\mathrm{HC}$ y, específicamente, la subcategoría Disciplinar, observamos que los profesores en formación inicial explicitan en sus respuestas 26 UA asociadas al código conceptual, de las que el 53,8 \% las utilizaron al inicio y el $46,2 \%$ al finalizar. Algunas de las UA fueron las siguientes: "El proceso de enseñanza no solo necesita un componente pedagógico, sino que él debe estar ligado a un componente científico" y "Con ayuda de la historia de la ciencia, podemos crear o ser parte de la creación de un concepto de científico como un ser cualquiera que se interesó en descubrir fenómenos, ampliar conceptos o innovar". En el caso del código actividad experimental, los futuros profesores expresan en sus respuestas $14 \mathrm{UA}$, de estas el 7,I \% las utilizaron al inicio y el $\mathbf{9 2 , 9} \%$ al finalizar. Algunas de las UA fueron: "Por medio de experimentación, exponiendo hipótesis, leyes y teorías, con el aporte, no solo de una persona, sino de una comunidad, donde se logre demostrar de alguna manera el fenómeno o experimento" y "La comprensión del modelo instrumental en el proceso de pasteurización incita a responder el cómo, por qué y para qué de esos instrumentos por medio del textos científico-históricos”. 
En la subcategoría Actividad social, los profesores en formación inicial emitieron 29 UA relacionadas con el código práctica educativa, de ellas, el $13,8 \%$ las expresaron al inicio y el $86,2 \%$ al final. Algunas de las UA acumulativa fueron las siguientes: "La historia de las ciencias proporciona los lineamientos, las estrategias y los parámetros que me permiten llevar a cabo en el acto educativo la enseñanza de las ciencias" y "Reflexionar en una enseñanza más flexible, con metodologías que centren la actuación en el alumno, para favorecer la metacognición, es decir, aprender a aprender". Mientras que en el código formación de profesores los futuros profesores expresan en sus respuestas 29 UA, de estas el $58,6 \%$ las utilizaron al inicio y el $41,4 \%$ al finalizar. Algunas de las UA fueron: "Estas son características muy necesarias en lo que es la formación en la ciencias y su enseñanza" y "Entender y enseñar la historia de la ciencia es un proceso que requiere la preparación del profesorado en historia y filosofía de las ciencias desde su formación”.

En la subcategoría Recurso, los profesores en formación inicial emitieron 23 UA relacionadas con el código uso de materiales, de ellas, el $13 \%$ las expresaron al inicio y el $87 \%$ al final. Algunas de las UA fueron las siguientes: "Ayuden a construir un conocimiento que sea diferente del que se plasma en los textos escolares, ya que en estos se incluyen conclusiones de la misma ciencia que no brinda suficiente contenido para analizar" y "La importancia que poseen las ilustraciones que utilizan los libros de texto de ciencias para la comprensión de su contenido". En cuanto al código formulación de preguntas, los participantes enunciaron II 2 UA y el $100 \%$ las identificamos al final.Algunas de las UA: “¿Qué tipo de neurona intervino en ello?”, “Piensa que ser alguien que cambie el giro de la ciencia u otra área es algo imposible?" y "Se realizará una socialización sobre el concepto y sus términos, y la importancia en la actualidad".

En la categoría Función de la historia de la ciencia, fue donde más UD elaboraron los profesores en formación inicial. Se logró identificar ideas relacionadas con los componentes desarrollados durante el semestre; por ejemplo, al finalizar, los participantes otorgaron un papel preponderante a la actividad experimental, la práctica educativa, el uso de materiales y la formulación de preguntas. En este último caso, las preguntas que formularon estaban distribuidas en los tres planos de análisis y desarrollo, es decir, instrumental operativo (preguntas centradas en el contenido, relaciones que lo caracterizan, las posibles so- luciones, estrategias, procedimientos, fórmulas, cálculos, gráficos, tablas de datos y axiomas), personal significativo (preguntas que indagan procesos $y$ estados personales, puntos de vista, creencias, experiencias personales y contextos cotidianos) y relacional social (preguntas que promueven interacción y socialización entre los estudiantes) (Labarrere, \& Quintanilla, 2002). La formulación de este tipo de preguntas incentivará a los estudiantes de educación que se apliquen, $y$ al desarrollo de competencias de pensamiento científico, como la explicación, la justificación y la argumentación (Muñoz et al., 20I7).

Se pudo evidenciar que los profesores en formación inicial solo relacionan la Función de la historia de la ciencia como aspecto conceptual, y no consideran la actividad experimental como aspecto fundamental en la etapa inicial. Sin embargo, en los resultados finales, se puede determinar un cambio al analizar la frecuencia de la concepción de la actividad experimental. Esperamos que durante su proceso de formación universitaria las asignaturas permitan adquirir experiencias para coaccionar la actividad experimental y la conceptual, y mejorar esa concepción de verlas desligadas.

Es importante que los futuros profesores de ciencia comprendan las funciones de la historia de la ciencia tanto en la práctica educativa como en la formación de profesores, ya que ofrece un panorama de las ciencias en el que se pueden determinar aspectos conceptuales, que desde una visión didáctica permitirán su enseñanza y aprendizaje. Además, esta metadisciplina adquiere valor en la medida en que incluye a los estudiantes como partícipes de la cultura, les permite destacar sucesos que permitieron la construcción de conocimiento científico, favorece la elaboración de nuevos modelos y proporciona lineamientos, estrategias y parámetros que permiten llevar a cabo la enseñanza de las ciencias.

En otras palabras, en la formación de profesores y en la práctica educativa, es necesario que los participantes conozcan, interpreten, exploren, indaguen e investiguen sobre casos históricos de la ciencia. Asimismo, se deben planificar actividades académicas escolares en las que se incorporen el uso de textos científico-históricos como herramientas que faciliten la comprensión de contenidos de la ciencia, la evaluación e innovación metodológica $y$, sobre todo, la función de la mujer en el desarrollo científico (Solsona, 2015). Esto conlleva la implementación de cambios en la estructura escolar, ya que se incorporarán 
los hallazgos obtenidos a partir de los estudios de caso históricos, que innovarán los procesos de construcción de conocimiento científico en el aula (Acevedo, GarcíaCarmona, \& Aragón-Méndez, 2017).

La historia de las ciencias como recurso y, específicamente, el uso de materiales tales como los libros de texto escolares permitirán mostrar en ellos el proceso y las dificultades por los que atravesó la investigación científica. De forma paralela, se debe continuar el diseño y la producción de materiales didácticos que la incorporen como un recurso para comprender el desarrollo del conocimiento científico (Cabrera, 20 I2; Muñoz Bello, \& Bertomeu, 2003; Quintanilla, \& Merino, 2008; Velasco, 2008).

Por último, el código conceptual mantuvo un margen equilibrado entre los resultados iniciales y finales del semestre; por ello, se puede establecer que estos son fundamentales para la comprensión de argumentos, teorías, conceptos y modelos de las ciencias por parte de los estudiantes. Se debe hacer énfasis en que estos contenidos no se deben continuar presentando de manera tradicional, es decir, como un listado, sino que se han de construir núcleos temáticos en los que se vislumbre los aportes de la historia de la ciencia y se adapten a las controversias científicas actuales en las que participan los profesores en formación inicial (Cabrera, 20 I6; Izquierdo, 2005).

Finalmente, en la categoría Aplicación de la HC, y concretamente la subcategoría Desarrollo, observamos que los profesores en formación inicial explicitan en sus respuestas 16 UA asociadas al código aplicación, de las que el $50 \%$ las utilizaron tanto al inicio como al final.Algunas de las UA fueron las siguientes:"Por los avances en las investigaciones científicas, ella ha logrado consolidarse como el punto central en el campo de la investigación, de ahí radica su importancia a nivel mundial" y "Los avances realizados, las distintas asociaciones identificadas y los términos usados son un reflejo de lo valioso que fue su trabajo para el desarrollo evolutivo del término simbiosis y los aportes tan significativos para el estudio de la biología".

Los profesores en formación inicial interpretan que, a partir de la historia de las ciencias, se puede identificar que la producción de conocimiento está relacionada con el desarrollo y avance de las ciencias, gracias a sus aportes a la sociedad, y porque permite plantear soluciones a problemas sociocientíficos del entorno social y ambiental, con las que se logre la estabilidad económica y el mejoramiento de la calidad de vida. Además, asumen que la producción de conocimiento está entendida como avances, estudios e investigaciones que contribuyen principalmente a la salud e innovación de campos médicos y forenses.

\section{Conclusiones}

De acuerdo con los hallazgos obtenidos, se logró identificar que las concepciones sobre historia de las ciencias que los profesores en formación inicial han elaborado corresponden a las visiones anacrónica y diacrónica, funciones como actividad social, recurso y disciplinar, y producción de conocimiento. En este sentido, se deben desarrollar e implementar propuestas didácticas que tengan componentes propios de la historia de las ciencias, que serán de mucha ayuda para la comprensión de asuntos científicos y contextuales, tanto para los profesores como para los estudiantes.

La implementación de propuestas didácticas enfocadas en la historia de las ciencias incentiva a los profesores en formación inicial al reconocimiento, la reflexión y el uso de componentes históricos en sus prácticas educativas. Las investigaciones de este tipo permiten reconocer los estados de las concepciones de estudiantes en formación, lo que es importante para futuras propuestas didácticas, para la reformulación de enfoques curriculares o para innovar o modificar planteamientos académicos. Para finalizar, se describen algunas de las conclusiones obtenidas a partir del análisis descriptivo de la investigación.

En la categoría Visión de la historia de las ciencias, se evidencia una transformación de ideas asociadas por los profesores en formación inicial, ya que, después de la propuesta didáctica, las UA relacionadas con el código acumulativo disminuyeron a 10 y las UA de valoración del contexto ascendieron a 27. La propuesta didáctica permitió mejorar el reconocimiento de los diferentes contextos que incurren en la construcción de los conocimientos y en la importancia e incidencia de estos, además, aleja la idea de construcción individualista y cronológica de la ciencia.

El análisis que se realizó en torno a la categoría Función de la historia de las ciencias estableció resultados fundamentales para las futuras investigaciones. Por ejemplo, las UA relacionadas con el código actividad experimental mostraron un cambio muy alentador para la investigación, pues inicialmente solo se había relacionado una UA, pero después de la propuesta didáctica aumentó a I3. La 
propuesta didáctica rescató la experimentación como un actor importante en la transformación de la construcción de conocimiento científico, y brindó herramientas a los profesores formación inicial que permiten el reconocimiento y uso de esta en las aulas de clase.

Del mismo modo, en la subcategoría Actividad social, las UA asociadas al código práctica educativa mostraron un desarrollo significativo, ya que inicialmente se relacionaron cuatro UA, pero luego de la práctica didáctica relacionaron 25. Esto permite concluir que el reconocimiento y uso de la historia de las ciencias en las prácticas educativas por parte de los profesores en formación inicial será importante como ejes estructuradores de los conocimientos en ciencia. Otro punto a resaltar es la subcategoría recurso, pues demuestra que finalmente los futuros profesores hacen mayor uso de la historia de las ciencias para la construcción de sus propuestas didácticas.

Finalmente, se destaca la importancia que los profesores en formación inicial dan a la formulación de preguntas como recurso en pro de la funcionalidad, ya que estas están asociadas a la construcción de conocimientos de ciencia, representado como código formulación en la fase final por una frecuencia de II 2 UA. Esto puede ser proyectado para que futuras investigaciones traigan a colación esa necesidad de estructura que pueden brindar las preguntas orientadoras enfocadas en la historia de las ciencias y los estados de desarrollo.

\section{Agradecimientos}

Los autores de este trabajo agradecen al Proyecto REDES I50I77 y a la Universidad del Valle la posibilidad de compartir y socializar este trabajo colaborativo. Además, declaramos que no tenemos ningún interés comercial o asociativo que presente un conflicto de intereses con el trabajo presentado.

\section{Referencias}

Acevedo-Díaz,J.A., García-Carmona,A., \& Aragón-Méndez, M. del M. (2017). Historia de la ciencia para enseñar naturaleza de la ciencia: una estrategia para la formación inicial del profesorado de ciencia. Educación Química, 28(3), 140 |46. http://dx.doi.org//0.1016/j.eq.2015.05.00 I
Álvarez, M. (2006). La historia de la ciencia en la formación del profesorado de ciencias. En M. Quintanilla Gatica, \& A.Adúriz-Bravo (Eds.), Enseñar ciencias en el nuevo milenio: retos y propuestas (pp. 239-256). Santiago de Chile, Chile: Pontificia Universidad Católica de Chile.

Bardin, L. (2002). Análisis de contenido. (3. . ed.). Madrid, España: Akal.

Boido, G., \& Lombardi, O. (20/2a). Las relaciones entre la historia y la filosofía de la ciencia (Primera parte). Exactamente, 49, 39.

Borrachero Cortés, A. B., Brígido Mero, M., \& Costillo Borrego, E. (20II). Concepciones sobre la ciencia de los alumnos del C.A. P. futuros profesores de Educación Secundaria. Revista de Educación Campo Abierto, 30(I), 63-82. Recuperado de http://revistas.ojs.es/index.php/campoabierto/article/download/572/510

Cabrera Castillo, H. G. (20/2). Análisis descriptivo sobre el concepto combustión en libros de texto universitarios. Revista Eureka sobre Enseñanza y Divulgación de las Ciencias, 9(3), 3। I-328.

Cabrera Castillo, H. G. (20I5). Los modos de representación de modelos en el curso Educación en Química con profesores en formación inicial en Ciencias Naturales. Revista Eureka sobre Enseñanza y Divulgación de las Ciencias, I2(3), 565-580.

Cabrera Castillo, H. G. (2016). Aportes a la enseñanza de la química a partir de un estudio histórico filosófico de la experimentación asociada a la combustión para profesores en formación inicial (Tesis de maestría, Universidad del Valle, Cali, Colombia).

Camacho,J.P., Cuéllar, L., Quintanilla, M., \& Orellana, M. (2006). Historia de la ciencia en la formación inicial de profesoras de educación infantil: una propuesta para el cambio conceptual. Santiago de Chile, Chile: Pontificia Universidad Católica de Chile.

Cuéllar, L., Quintanilla, M., \& Camacho, J. P. (2008). Introducción de la historia de la química en la formación docente: aportes para un debate teórico y campo. Actes d'Història de la Ciència i de la Tècnica, I (2), 109-I 17. http://dx.doi. org//0.2436/20.2006.01.64

Echeverría, J. (1998). Filosofia de la ciencia. Madrid, España:Akal.

Fernández, I., Gil, D., Carrascosa, J., Cachapuz, A., \& Praia, J. (2002).Visiones deformadas de la ciencia transmitidas por la enseñanza. Enseñanza de las Ciencias, 20(3), 477-488. 
Flick, U. (2004). Introducción a la investigación cualitativa. Madrid, España: Morata.

Gallego Torres, A. P., \& Gallego Badillo, R. (2007). Historia, epistemología y didáctica de las ciencias: unas relaciones necesarias. Ciência \& Educação, /3(I), 85-98. http://dx.doi. org/I0.1590/SI516-73132007000100006

Gibbs, G. (20I2). El análisis de datos en investigación cualitativa. Madrid, España: Morata.

Heering, P., \& Wittje, R. (20I2). An historical perspective on instruments and experiments in science education. Science \& Education, 2 I (2), I 5 I - I55. http://dx.doi.org/ I0. I007/ s। I I9I-010-9334-z

Hernández, M. (20I I). Elementos para la enseñanza del concepto homeostasis presentes desde el estudio de su desarrollo histórico (Tesis de grado, Universidad del Valle, Cali, Colombia).

Hernández Sampieri, R., Fernández Collado, C. y Baptista Lucio, P. (2008). Metodología de la investigación. (4. ${ }^{\text {a ed.). Ciu- }}$ dad de México, México: McGraw-Hill.

Holton, G. (1995): Understanding The History of Science. En: Einstein, History, and Others Passions.Woodbury, N.Y.:American Institute of Physics 185-201.

Izquierdo i Aymerich, M. (2005). Hacia una teoría de los contenidos escolares. Enseñanza de las Ciencias, 23(I), I I I122.

Izquierdo i Aymerich, M. (20I4). Pasado y presente de la química: su función didáctica. En C. Merino, M. Arellano, \& A. Adúriz-Bravo (Eds.), Avances en didáctica de la química: modelos y lenguajes (pp. 13-36).Valparaiso, Chile: Ediciones Universitarias de Valparaiso de la Universidad Católica de Valparaiso.

Izquierdo i Aymerich, M., García-Martínez, A., Quintanilla, M., \& Adúriz-Bravo, A. (2016). Historia, filosofia y didáctica de las ciencias: aportes para la formación del profesorado de ciencias. Bogotá, Colombia: Universidad Distrital Francisco José de Caldas.

Justi, R. (2006). La enseñanza de ciencias basada en la elaboración de modelos. Enseñanza de las Ciencias, 24(2), I73-184. Recuperado de http://www.cneq.unam.mx/cursos_diplomados/diplomados/medio_superior/SEIEM/Ia/0I/00/02_ material/la_generacion/mod8/archivos/Justi, 2006.pdf

Kragh, H. (1990). Introducción a la historia de la ciencia. Barcelona, España: Crítica.

Labarrere, A., \& Quintanilla, M. (2002). La solución de problemas científicos en el aula: reflexiones desde los planes de análisis y desarrollo. Pensamiento Educativo, 30(I), I 2 I 137. Recuperado de http://pensamientoeducativo.uc.cl/ files/journals/2/articles/2 I6/public/2 I6-509-I-PB.pdf
Latorre,A., del Rinón, D., \& Arnal,J. (1996). Bases metodológicas de la investigación educativa. Barcelona, España: Gràfiques.

Matthews, M. R. (1994). Historia, filosofía y enseñanza de las ciencias: la aproximación actual. Enseñanza de las Ciencias, I2(2), 255-277.

Muñoz, F., Valencia, E., \& Cabrera Castillo, H. G. (2017). Situaciones científicas escolares problematizadoras a partir del análisis del experimento $\mathrm{V}$ de Robert Boyle. Revista Eureka sobre Enseñanza y Divulgación de las Ciencias, I4(I), II5-125.

Muñoz Bello, R., \& Bertomeu Sánchez, J. R. (2003). La historia de la ciencia en los libros de texto: las hipótesis de Avogadro. Enseñanza de las Ciencias, 2 I (I), I47-I59.

Padilla, K., Furió, C., \& Azcona, R. (2005). Las visiones deformadas de la ciencia en la enseñanza universitaria de los conceptos de cantidad de sustancia y mol. Enseñanza de las Ciencias (Número Extra VII Congreso), I-5.

Quintanilla Gatica, M. (2006a). Historia de la ciencia, ciudadanía y valores: claves de una orientación realista pragmática de la enseñanza de las ciencias. Revista Educación y Pedagogía, 23(45), 7-23.

Quintanilla Gatica, M. (2006b). La emergencia de la historia de la química en la formación del profesorado. Ponencia presentada en X Encuentros de Educación Química,Talca, Chile.

Quintanilla Gatica, M., Astroza, V., De la Fuente, R., Camacho, J. P., \& Cuéllar, L. (2006). Imagen de las metaciencias en la formación inicial de profesores de EGB. Santiago de Chile, Chile: Pontificia Universidad Católica de Chile.

Quintanilla Gatica, M., Izquierdo, M., \& Adúriz-Bravo,A. (2005). Avances en la construcción de marcos teóricos para incorporar la historia de la ciencia en la formación inicial del profesorado de ciencias naturales. Enseñanza de las Ciencias (Número Extra), I-4.

Quintanilla Gatica, M., Izquierdo, M., \& Adúriz-Bravo,A. (2007). Discusión en torno a un modelo para introducir la historia de la ciencia en la formación inicial del profesorado de ciencias. En M. Izquierdo, A. Caamaño, \& M. Quintanilla Gatica (Eds.), Investigar en la enseñanza de la química. Nuevos horizontes: contextualizar y modelizar (pp. 173-196). Barcelona, España: Universidad Autónoma de Barcelona.

Quintanilla Gatica, M., Labarrere, A., Santos, M., Cadiz, J., Cuéllar, L., Saffer, G., \& Camacho, J. P. (2006). Elaboración, validación y aplicación preliminar de un cuestionario sobre ideas acerca de la imagen de ciencia y educación científica de profesores en servicio. Santiago de Chile, Chile: Pontificia Universidad Católica de Chile. 
Quintanilla Gatica, M., \& Merino, C. (2008). Elaborar unidades didácticas incorporando la historia de la ciencia. En $\mathrm{C}$. Merino, A. Gómez, \& A. Adúriz-Bravo (Eds.), Áreas y estrategias de investigación en la didáctica de las ciencias experimentales (Pp. I49-168). Barcelona, España: Universidad Autónoma de Barcelona.

Solsona Pairó, N. (1997). L'emergència de la interpretació dels fenòmens químics. Barcelona, España: Universidad Autonoma de Barcelona.

Solsona Pairó, N. (20I5). Análisis de las estrategias de autorización de mujeres científicas en la llustración. Revista Física y Cultura, 9, 25-40.

Urzúa Hernández, M. del C., \& Rodríguez-Pineda, D. P. (20I5). Visiones sobre ciencia de estudiantes universitarios en el contexto del laboratorio de microbiología y su relación con la historia. Bio-grafía: Escritos sobre la Biología y su Enseñanza (Número extraordinario), 194-202. http://dx.doi. org//0.17227/2027/034.vol.0num.0bio-grafial 94.202
Valencia, E., Muñoz, F., \& Cabrera, H. G. (20l4). Análisis de texto histórico desde una mirada educativa: el caso del experimento $\vee$ de Robert Boyle. En Tercera Conferencia Latinoamericana del Grupo Internacional de Historia, Filosofía y Enseñanza de las Ciencias (PP. I-10). Santiago de Chile, Chile: Bellaterra.

Velasco, J. (2008). Historia de la ciencia y enfoque historiográfico en libros de Ciencias Biológicas de Educación Básica y Educación Media Diversificada Profesional en Venezuela. Revista de Investigación, 32(64), 63-84.

Zuluaga, C. (2012). Historia y epistemología de la química en la selección y secuenciación de contenidos: la construcción del concepto de átomo. Revista EDUCyT, 5, 95-I 16. 